\title{
Fuzzy Linear and Nonlinear Integral Equations: Numerical Methods
}

\author{
Reza Ezzati, ${ }^{1}$ Soheil Salahshour, ${ }^{2}$ Ronald R. Yager, ${ }^{3}$ and Morteza Khodabin ${ }^{1}$ \\ ${ }^{1}$ Department of Mathematics, College of Basic Sciences, Islamic Azad University, Karaj Branch, Alborz, Iran \\ ${ }^{2}$ Young Researchers and Elite Club, Mobarakeh Branch, Islamic Azad University, Mobarakeh, Iran \\ ${ }^{3}$ Machine Intelligence Institute, Iona College, New Rochelle, NY 10801, USA \\ Correspondence should be addressed to Reza Ezzati; ezati@kiau.ac.ir
}

Received 17 November 2014; Accepted 17 November 2014; Published 22 December 2014

Copyright (C) 2014 Reza Ezzati et al. This is an open access article distributed under the Creative Commons Attribution License, which permits unrestricted use, distribution, and reproduction in any medium, provided the original work is properly cited.

Integral equations are one of the most useful mathematical tools in both pure and applied mathematics. They have enormous applications in many real problems. Many initial and boundary value problems associated with ordinary differential equation (ODE) and partial differential equation (PDE) can be transformed into problems of solving some approximate integral equations.

Indeed, modeling such problems using integral equations with the exact parameters is not only easy but also impossible in the real problems. For this purpose, one way is using some uncertainty measures for handling such lack of information. One of the most and recent approaches is using Zadeh's fuzzy concept. So, instead of using deterministic models, we provide fuzzy integral equations of both linear and nonlinear forms.

In fact, obtaining the exact solutions of such fuzzy integral equations is not possible in all cases because of the inherited restrictions form application of fuzzy concepts in these problems. So, in this special issue, we intend to consider the numerical methods to solve fuzzy integral equations and the related topics with real applications. These topics include fuzzy linear and nonlinear integral equations with numerical methods, investigating the convergence, stability, and consistency of numerical approaches, numerically modeling the real problems associated with numerical methods, considering the differences between deterministic and fuzzy numerical methods to solve fuzzy integral equations, numerically solving fuzzy differential equations of arbitrary order using the equivalence fuzzy integral equations, obtaining some approximations of the solutions via ranking approaches, and applications in real-world problems with numerical techniques.

Our special issue contains few papers in which different numerical techniques are employed. The paper "A simplified Milstein scheme for SPDEs with multiplicative noise" replaces the exponential term with a Padé approximation of order 1 and denotes the resulting scheme by simplified Milstein scheme. The paper "On properties of pseudointegrals based on pseudoaddition decomposable measures" discussed pseudointegrals based on a pseudoaddition decomposable measure. Particularly, the definition of the pseudointegral for a measurable function based on a strict pseudoaddition decomposable measure by generalizing the definition of the pseudointegral of a bounded measurable function was stated. The paper "Quadrature rules and iterative method for numerical solution of two-dimensional fuzzy integral equations" introduced some generalized quadrature rules to approximate two-dimensional, Henstock integral of fuzzynumber-valued functions. Also, it gave error bounds for mappings of bounded variation in terms of uniform modulus of continuity. Moreover, it proposed an iterative procedure based on quadrature formula to solve two-dimensional linear fuzzy Fredholm integral equations of the second kind (2DFFLIE2) and presented the error estimation of the proposed method. The paper "On solution of integrodifferential equation with delay parameter by Sinc basis functions" is considered. For this purpose, a numerical solution is obtained for an integrodifferential equation with an integral boundary 
condition and delay parameter. This type of problems arises in mathematical physics, mechanics, population growth, and other fields of physics and mathematical chemistry. Then, convergence of this approach is discussed by presenting a theorem which gives exponential type convergence rate and guarantees the accuracy of that. The paper " $A$ new reconstruction of variational iteration method and its application to nonlinear Volterra integrodifferential equations" is proposed. Indeed, it reconstructed the variational iteration method, that is, the so-called parametric iteration method (PIM). The proposed method was applied for solving nonlinear Volterra integrodifferential equations (NVIDEs). The paper "Approximating the solution of the linear and nonlinear fuzzy Volterra integrodifferential equations using expansion method" is considered. To this end, it introduced an innovative method applying power series to solve numerically the linear and nonlinear fuzzy integrodifferential equation systems.

We hope the papers published in this special issue will provide a useful guide to a large community of researchers and will give way to development of new innovative theories and numerical approaches in the fields of modeling and approximating fuzzy integral equations and the related topics.

\section{Acknowledgments}

We thank all the authors and the honorable reviewers who contributed to this special issue.

Reza Ezzati

Soheil Salahshour

Ronald R. Yager

Morteza Khodabin 


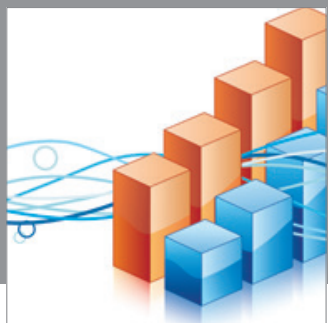

Advances in

Operations Research

mansans

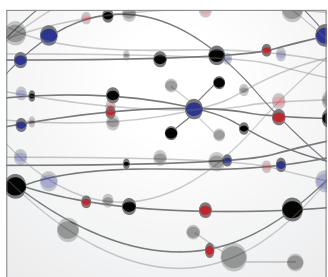

The Scientific World Journal
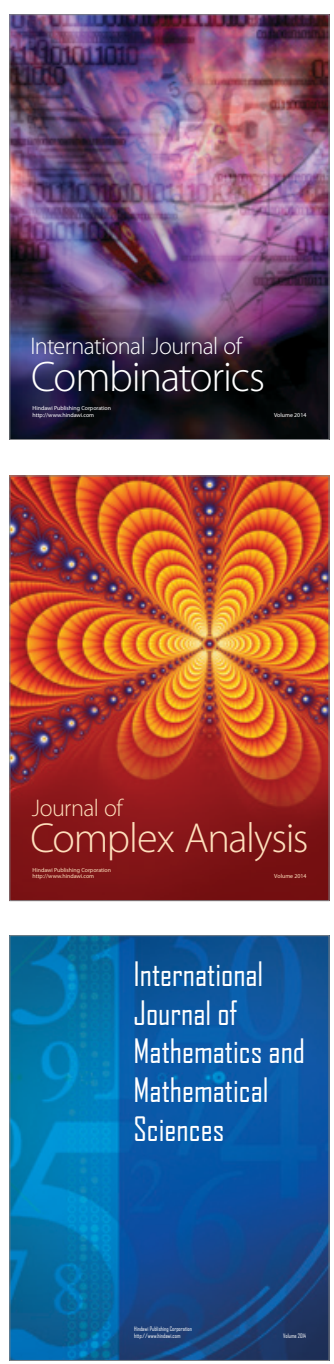
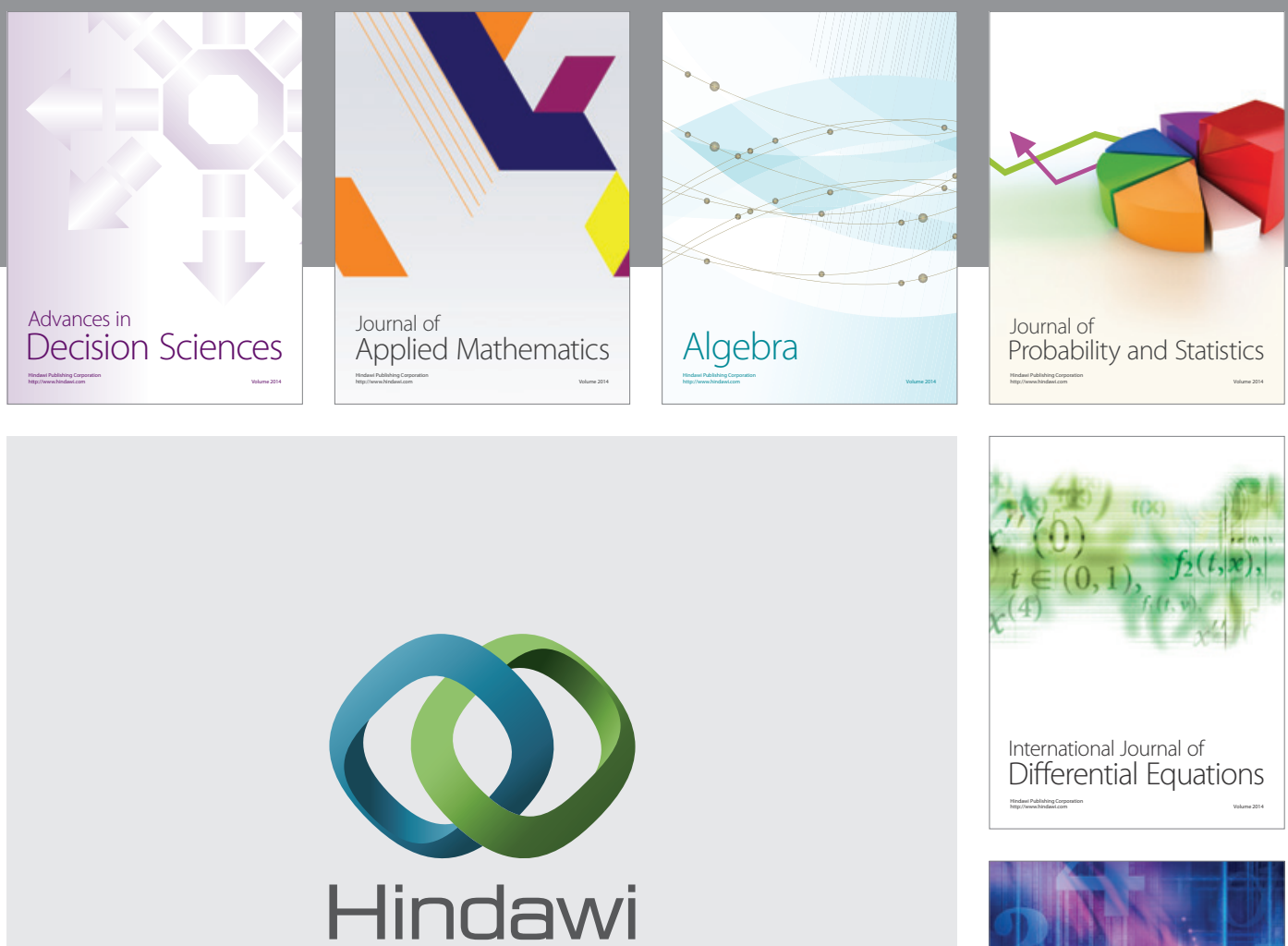

Submit your manuscripts at http://www.hindawi.com
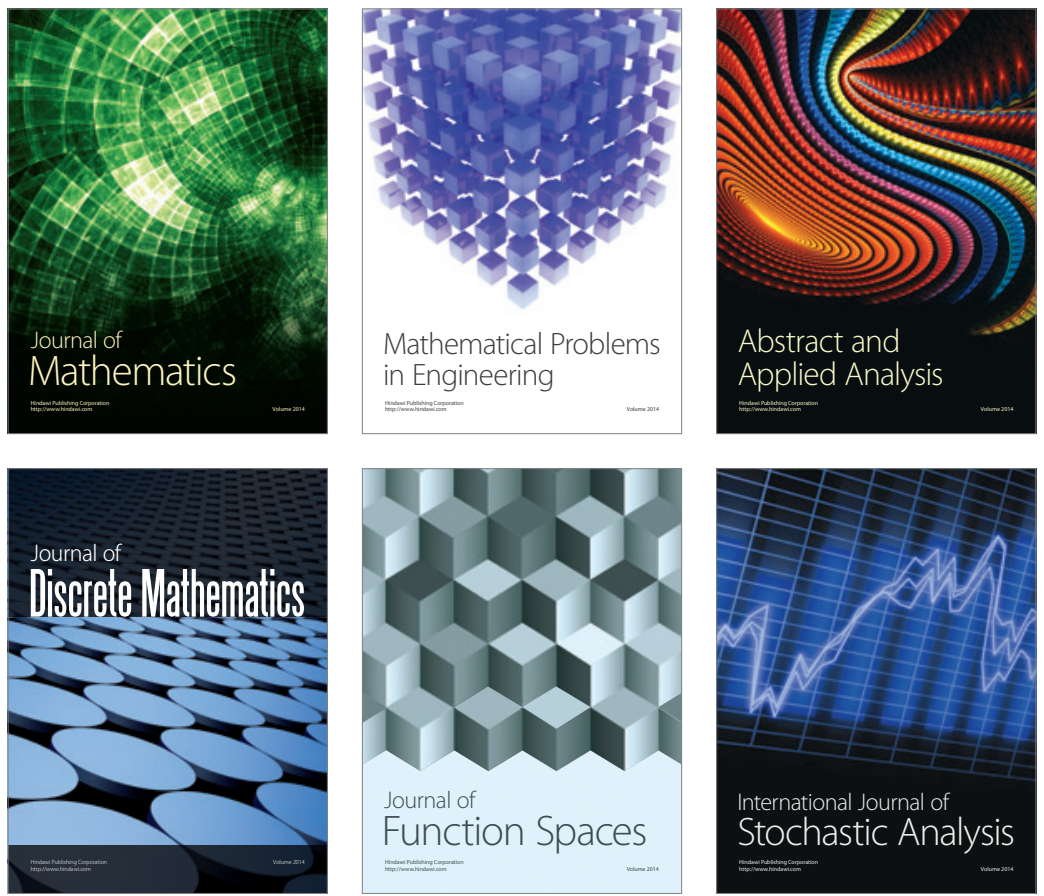

Journal of

Function Spaces

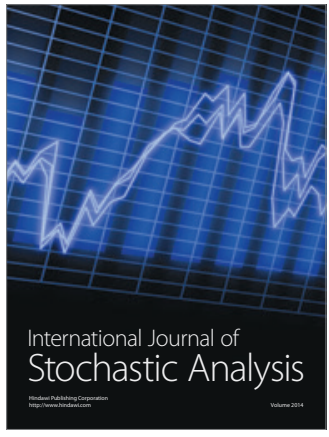

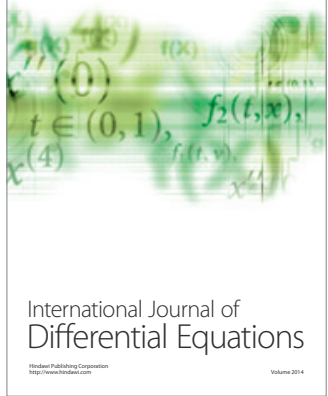
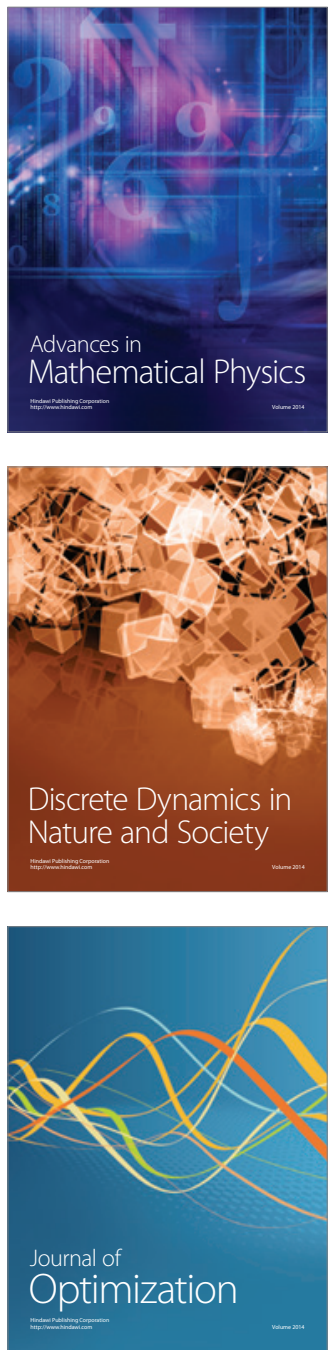